CARDIOVASCULAR MEDICINE

\title{
Acute improvement of atrial mechanical stunning after electrical cardioversion of persistent atrial fibrillation: comparison between biatrial and single atrial pacing
}

\author{
M Takagi, A Doi, N Shirai, K Hirata, Y Takemoto, K Takeuchi, J Yoshikawa
}

Heart 2005;91:58-63. doi: 10.1136/hrt.2003.032334

\begin{abstract}
Objective: To evaluate the acute effects of atrial pacing at different pacing sites on mechanical stunning after cardioversion of atrial fibrillation (AF).

Setting: Tertiary referral centre.

Patients: 20 patients with persistent AF were studied.

Interventions: Spontaneous echo contrast (SEC), left atrial appendage emptying velocity (LAAEV), and left atrial appendage emptying fraction (LAAEF) were assessed by transoesophageal echocardiography (TOE) during $\mathrm{AF}$, after conversion to sinus rhythm, and during atrial pacing from the right atrial appendage, left lateral atrium, and both atria simultaneously. Transmitral inflow velocity of the atrial wave (TMIF-A) by TOE and the maximum $P$ wave duration in 12 lead ECG were also measured during sinus rhythm and atrial pacing. Main outcome measures: Comparison of atrial mechanical function and P wave duration in 12 lead ECG during atrial pacing from different sites after cardioversion of AF.

Results: Compared with sinus rhythm, atrial pacing at 80 beats/min increased LAAEV from mean (SD) $14.6(10.1)$ to $33.4(19.8) \mathrm{cm} / \mathrm{s}(\mathrm{p}=0.001)$, LAAEF from $13.8(8.5)$ to $32.1(11.2) \%(\mathrm{p}<0.001)$, and TMIF-A from $24.6(11.9)$ to $45.6(21.0) \mathrm{cm} / \mathrm{s}(\mathrm{p}<0.001)$ and reduced SEC grade from $2.6(1.0)$ to 1.6 (0.9) $(p<0.001)$. These effects had a positive force-frequency relation. Biatrial pacing produced the shortest $P$ wave duration and resulted in the most significant improvement in atrial function (LAAEV, 33.2 (19.3) v $53.7(23.9) \mathrm{cm} / \mathrm{s}, \mathrm{p}=0.0001$; LAAEF, 31.9 (11.1) v 46.2 (12.6)\%, p $<0.0001$; TMIF-A, 37.7 (18.3) v $54.1(21.2) \mathrm{cm} / \mathrm{s}, \mathrm{p}<0.001$; SEC grade, $1.4(1.1) \vee 0.8(0.9), p=0.001$, right atrial appendage versus biatrial pacing).

Conclusions: Atrial pacing at increased rates can improve atrial mechanical function after cardioversion of persistent AF. Biatrial pacing may be the most effective technique to reverse atrial mechanical stunning.
\end{abstract}

See end of article for authors' affiliations

Correspondence to:

Dr Masahiko Takagi,

Department of Internal

Medicine and Cardiology,

Osaka City University

Graduate School of

Medicine, 1-4-3

Asahimachi, Abeno-ku,

Osaka 5458585, Japan;

m7424580@msic.med.

osaka-cu.ac.jp

Accepted 2 April 2004
A transient decrease in atrial mechanical function, referred to as atrial mechanical stunning, is a well documented phenomenon after cardioversion of atrial fibrillation (AF). ${ }^{1-4}$ Atrial mechanical stunning is thought to be one of the mechanisms responsible for the increased risk of thromboembolic complications after cardioversion of AF. ${ }^{56}$ A number of transthoracic and transoesophageal echocardiography (TOE) studies have shown that after cardioversion the mechanical function of the left atrium and left atrial appendage (LAA) is reduced, resulting in lower LAA blood flow velocities, which in turn predisposes patients to the formation of new thrombi. ${ }^{1-12}$ The atrial mechanical stunning is thought to be related to the properties of the preceding arrhythmia rather than the mode of cardioversion and can be considered a form of tachycardia induced cardiomyopathy. ${ }^{13}$ Sanders and colleagues ${ }^{14}{ }_{15}^{15}$ showed that atrial mechanical stunning after cardioversion of chronic atrial flutter and short duration $\mathrm{AF}$ can be reversed both by atrial pacing and through the administration of either isoproterenol or calcium. However, both the effects of atrial pacing and the effects of varying the pacing sites and rates on atrial stunning after cardioversion of AF have not been evaluated. This study aimed to determine, by using TOE, the acute effects of atrial pacing and the effects of varying the pacing sites and rates on atrial mechanical function just after electrical cardioversion of persistent AF.

\section{METHODS}

\section{Patients}

We studied 20 consecutive patients ( 13 men, mean (SD) age 57 (15) years) who had persistent AF between 3-6 months in duration and underwent electrical cardioversion and TOE to rule out the presence of left atrial thrombi (table 1). All patients gave written informed consent to the study, which was approved by Clinical Research Ethics Committee of the Osaka City University.

All patients were treated with warfarin and controlled to have an international normalised ratio between 2-2.5 for three weeks before cardioversion. All antiarrhythmic drugs were continued before and after cardioversion.

\section{Echocardiographic analysis}

A viscous 2\% lidocaine solution was used for oropharyngeal anaesthesia and propofol was used for sedation. TOE was recorded with a $6 \mathrm{MHz}$ multiplane probe connected to an Acuson Sequoia ultrasound system (Acuson Corporation, Mountain View, California, USA).

Before cardioversion, LAA was scanned in planes to establish the angle for obtaining maximum LAA areas. The mitral valve was also scanned on a four chamber image to establish the angle at which the maximum transmitral flow could be obtained. During cardioversion, the probe was left in place and the echocardiograph disconnected. After cardioversion, the resultant angles were used for subsequent analysis.

LAA emptying velocity (LAAEV) was assessed by pulsed Doppler echocardiography by placing the sample volume

Abbreviations: AF, atrial fibrillation; LAA, left atrial appendage; LAAEF, left atrial appendage emptying fraction; LAAEV, left atrial appendage emptying velocity; RAA, right atrial appendage; SEC, spontaneous echo contrast; TMIF-A, transmitral inflow velocity of the atrial wave; TOE, transoesophageal echocardiography 


\begin{tabular}{|c|c|c|c|c|c|c|c|c|}
\hline $\begin{array}{l}\text { Patient } \\
\text { number }\end{array}$ & Age (years) & Sex & $\begin{array}{l}\text { Associated heart } \\
\text { disease }\end{array}$ & $\begin{array}{l}\text { Duration of AF } \\
\text { (months) }\end{array}$ & LVEF (\%) & $\begin{array}{l}\text { Left atrial } \\
\text { diameter }(\mathrm{mm})\end{array}$ & $\begin{array}{l}\text { SEC grade } \\
\text { during AF }\end{array}$ & $\begin{array}{l}\text { Sinus rate after } \\
\text { cardioversion (beats } / \mathrm{min} \text { ) }\end{array}$ \\
\hline 1 & 70 & $\mathrm{~F}$ & None & 3 & 56 & 44 & 1 & 70 \\
\hline 2 & 51 & $M$ & HHD & 5 & 55 & 49 & 2 & 38 \\
\hline 3 & 74 & $M$ & None & 3 & 59 & 35 & 1 & 66 \\
\hline 4 & 46 & M & DCM & 4 & 42 & 42 & 1 & 72 \\
\hline 5 & 76 & $\mathrm{~F}$ & None & 3 & 64 & 52 & 2 & 41 \\
\hline 6 & 58 & M & None & 3 & 58 & 41 & 1 & 76 \\
\hline 7 & 77 & M & DCM & 3 & 25 & 52 & 4 & 73 \\
\hline 8 & 58 & M & HHD & 3 & 44 & 38 & 1 & 66 \\
\hline 9 & 53 & $\mathrm{~F}$ & DCM & 5 & 37 & 55 & 2 & 72 \\
\hline 10 & 58 & $\mathrm{~F}$ & OMI & 3 & 31 & 53 & 1 & 41 \\
\hline 11 & 17 & M & None & 6 & 57 & 39 & 0 & 78 \\
\hline 12 & 51 & M & None & 6 & 70 & 38 & 0 & 76 \\
\hline 13 & 67 & M & DCM & 6 & 28 & 47 & 3 & 75 \\
\hline 14 & 34 & M & None & 3 & 60 & 43 & 1 & 74 \\
\hline 15 & 62 & $\mathrm{~F}$ & None & 3 & 55 & 46 & 4 & 64 \\
\hline 16 & 48 & M & $\mathrm{HCM}$ & 3 & 55 & 50 & 1 & 62 \\
\hline 17 & 52 & $M$ & HHD & 5 & 65 & 40 & 0 & 70 \\
\hline 18 & 68 & $\mathrm{~F}$ & OMI & 3 & 42 & 49 & 1 & 48 \\
\hline 19 & 56 & $\mathrm{~F}$ & None & 4 & 62 & 38 & 0 & 76 \\
\hline 20 & 70 & M & DCM & 5 & 32 & 52 & 2 & 55 \\
\hline
\end{tabular}

$A F$, atrial fibrillation; DCM, dilated cardiomyopathy; F, female; HCM, hypertrophic cardiomyopathy; HHD, hypertensive heart disease; LVEF, left ventricular ejection fraction; $M$, male; OMI, old myocardial infarction; SEC, spontaneous echo contrast.

$1.5 \mathrm{~cm}$ into the entrance of the LAA. Maximum and minimum LAA areas were measured by planimetry, and LAA emptying fraction (LAAEF) was calculated from the formula LAAEF $=$ (maximum LAA area - minimum LAA area/maximum LAA area). Left atrial function was assessed from pulsed Doppler interrogation of transmitral inflow by
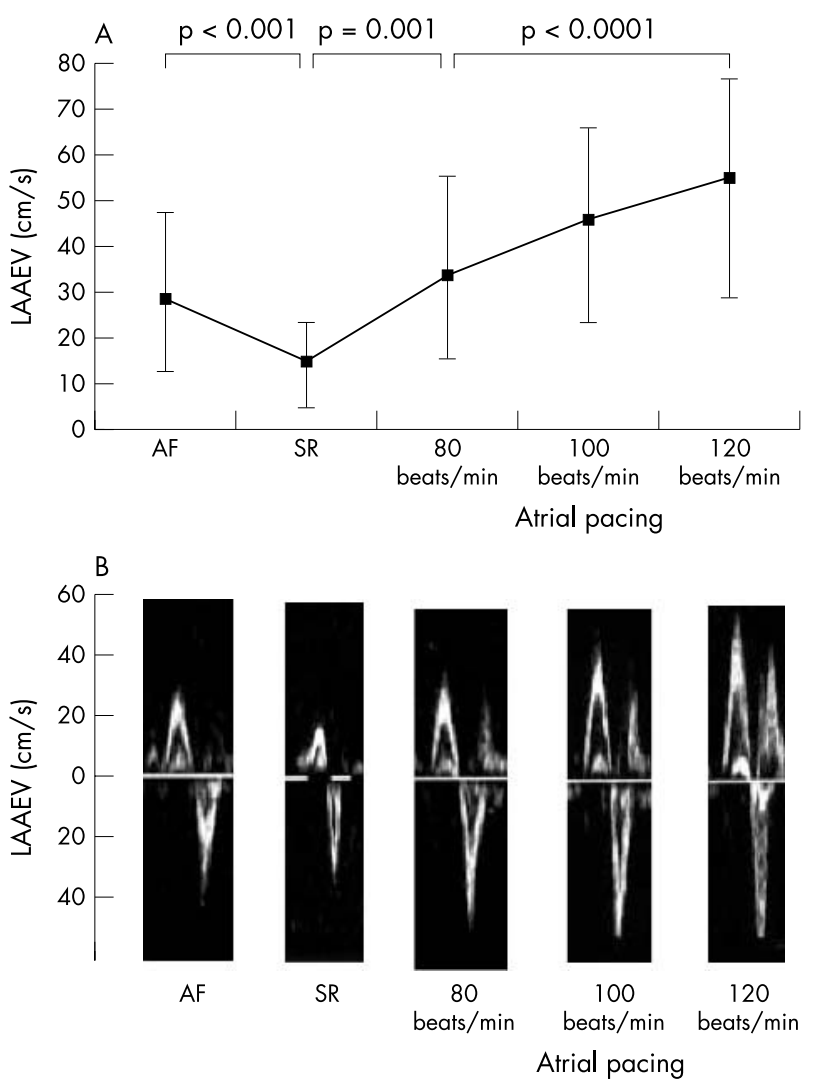

Figure 1 (A) Left atrial appendage emptying velocity (LAAEV) during atrial fibrillation (AF), sinus rhythm (SR) after cardioversion, and atrial pacing at 80,100 , and 120 beats/min. (B) Representative LAAEV. placing the sample volume at the leaflet tips, and the transmitral inflow velocity of the atrial wave (TMIF-A) was measured.

Spontaneous echo contrast (SEC) was defined as dynamic intracavity echoes of a swirling pattern distinct from white noise artefact. Gain settings were sequentially reduced to exclude white noise artefact. The degree of SEC was graded from 0 to 4 according to previously published criteria ${ }^{16}: 0$, none (absence of echogenicity); 1+, mild (minimum echogenicity located in the LAA or sparsely distributed in the main cavity of the left atrium); $2+$, mild to moderate (more dense swirling pattern than $1+$ but with similar distribution); $3+$, moderate (dense swirling pattern in the LAA, generally associated with somewhat lesser intensity in the main cavity); 4+, severe (intense echodensity and very slow swirling patterns in the LAA, usually with similar density in the main cavity).

These parameters were measured independently off line by two experienced observers. Three consecutive measurements taken during sinus rhythm after cardioversion and each atrial pacing were averaged. LAAEV, LAAEF, and SEC were also measured during $\mathrm{AF}$, and six consecutive measurements were averaged.

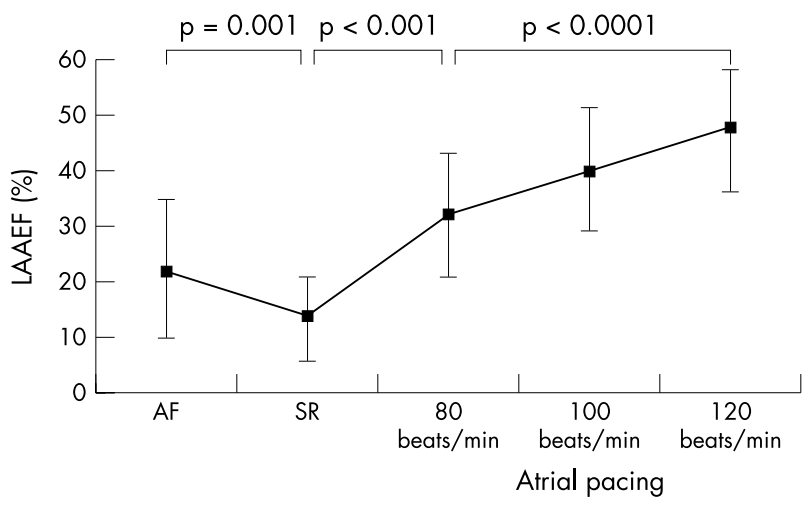

Figure 2 Left atrial appendage emptying fraction (LAAEF) during $A F$, SR after cardioversion, and atrial pacing at 80,100 , and 120 beats/ min. 

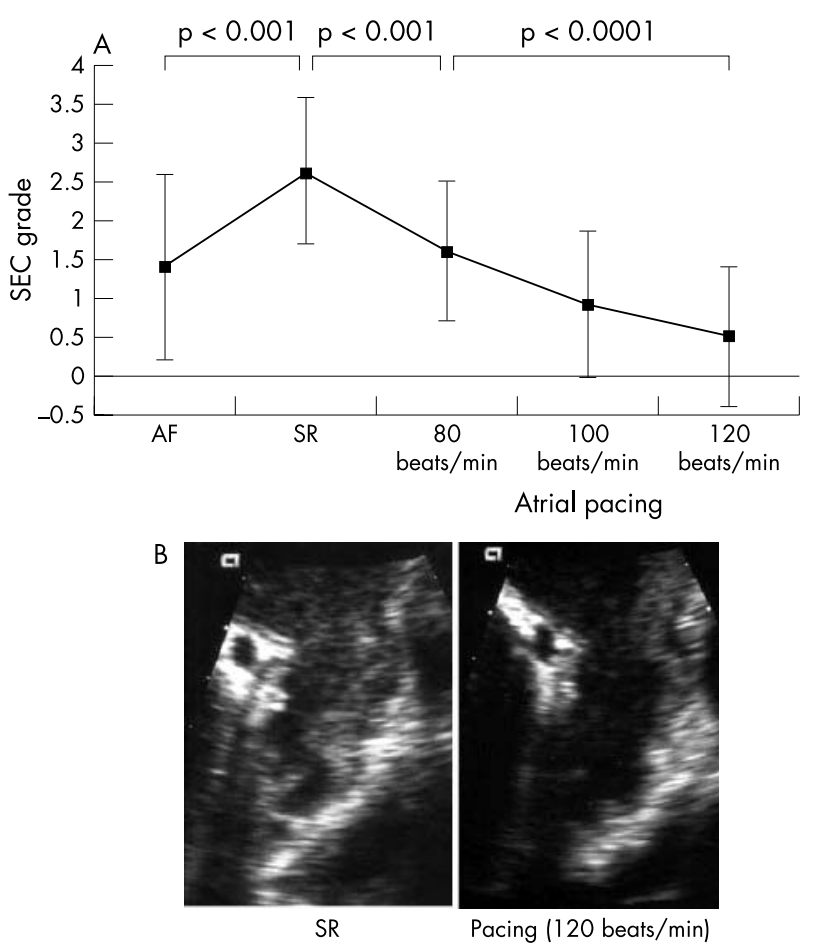

Figure 3 (A) Spontaneous echo contrast (SEC) grade during $A F, S R$ after cardioversion, and atrial pacing at 80,100 , and 120 beats $/ \mathrm{min}$. (B) Representative SEC during SR and pacing at 120 beats $/ \mathrm{min}$.

\section{Pacing protocol}

Before the initial TOE evaluation during AF, a 6 French steerable quadripolar electrode catheter with $2.5 \mathrm{~mm}$ interelectrode spacing (Steerocath Dx, EP Technologies Inc, San Jose, California, USA) was introduced percutaneously through the femoral vein and positioned in the right atrial appendage (RAA) under fluoroscopic guidance. A 5 French decapolar catheter with $2 \mathrm{~mm}$ interelectrode spacing (Torqr, Medtronic Inc, Minneapolis, Minnesota, USA) was placed into the left lateral site of the coronary sinus. After conversion to sinus rhythm, bipolar pacing at 80 beats/min presented in random order from the RAA, coronary sinus, and both atria simultaneously was performed at twice the diastolic threshold with a pulse duration of 2 ms delivered from a programmable stimulator (SEC-3120, Nihon Kohden, Tokyo, Japan). In 18 of 20 patients, who had no second degree atrioventricular blocks at 100 beats/min or 120 beats/ min, also underwent randomly presented bipolar pacing at 100 beats/min and 120 beats/min from the RAA, coronary sinus, and both atria. Surface 12 lead ECG and intracardiac bipolar electrograms were filtered from 30 to $500 \mathrm{~Hz}$ and recorded on a computer based digital amplifier and recorder system with optical disk storage. The maximum $\mathrm{P}$ wave duration was measured with computer assisted calipers at a sweep speed of $200 \mathrm{~mm} / \mathrm{s}$. The onset of the $\mathrm{P}$ wave was defined as the point of the first visible upward departure of the trace from the bottom of the baseline for the positive waves and as the point of the first downward departure from the top of the baseline for negative waves. The return of the baseline to the bottom of the trace in positive waves and to the top of the trace in negative waves was considered to be the end of the $\mathrm{P}$ wave. Maximum $\mathrm{P}$ wave duration during pacing was determined as the duration from the pacing spike to the end of the $\mathrm{P}$ wave in the same lead where the maximum $\mathrm{P}$ wave duration was found during sinus rhythm.
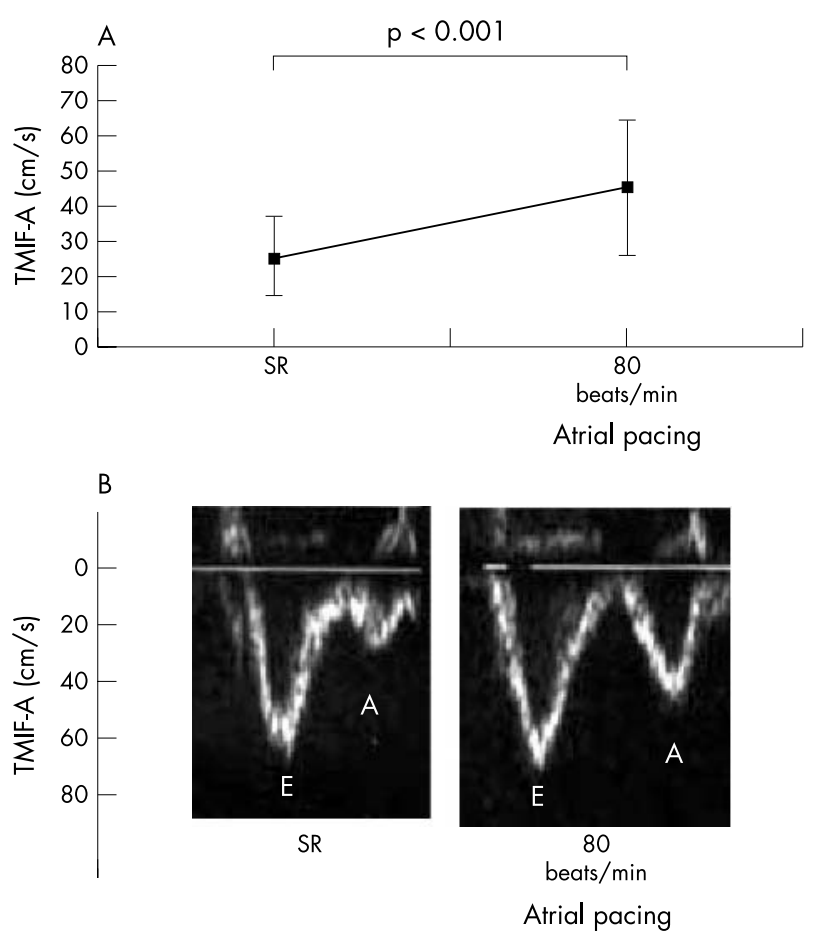

Figure 4 (A) Transmitral inflow velocity of the atrial wave (TMIF-A) during SR after cardioversion and atrial pacing at 80 beats $/ \mathrm{min}$. (B) Representative TMIF-A during SR and pacing at 80 beats $/ \mathrm{min}$. $A$, atrial filling wave; $E$, early filling wave.

\section{Cardioversion protocol}

After completion of the TOE measurements during AF, all patients underwent transthoracic electrical cardioversion under deep sedation. Sinus rhythm was restored by $100-$ $200 \mathrm{~J}$ shocks in all patients. No patient was found to have either a left atrial thrombus or thromboembolic complications before and after cardioversion.

\section{Study protocol}

In all patients, LAAEV, LAAEF, and SEC were measured during $\mathrm{AF}$, sinus rhythm after cardioversion, and atrial pacing from the RAA, coronary sinus, and both atria at 80 beats/min. TMIF-A was measured during sinus rhythm and atrial pacing. In 18 of 20 patients, LAAEV, LAAEF, and SEC were also measured during atrial pacing from the RAA, coronary sinus, and both atria at 100 beats/min and 120 beats/min to confirm a positive force-frequency relation.

These parameters were determined 10 minutes after conversion to sinus rhythm and 10 minutes after the start of atrial pacing from each site and at each rate. The interval between pacing was five minutes to avoid recording transitional results.

\section{Statistical analysis}

All variables are reported as mean (SD). Results during AF, sinus rhythm, and each atrial pacing at 80 beats/min, and the effects of pacing rate and pacing site were compared with nested analysis of variance with Scheffe's test. The interobserver and intraobserver variability were assessed according to the Bland and Altman method. Values of $\mathrm{p}<0.05$ were considered significant.

\section{RESULTS}

\section{Characteristics of patients with persistent AF}

Of the 20 patients, five had dilated cardiomyopathy, three had hypertensive heart disease, two had previous myocardial infarction, one had hypertrophic cardiomyopathy, and the 


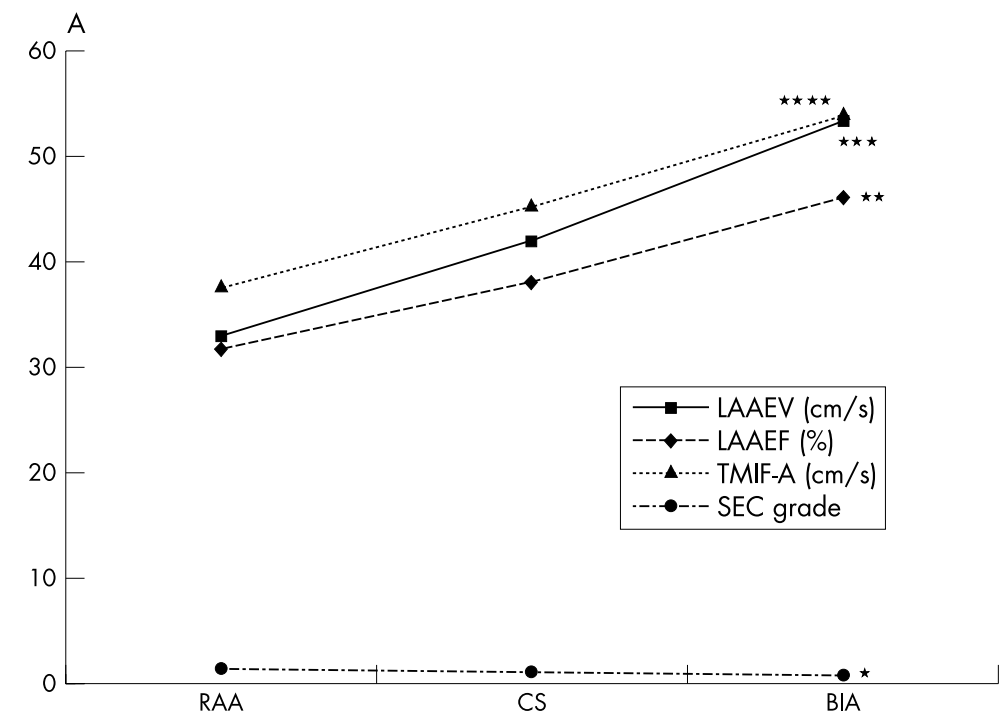

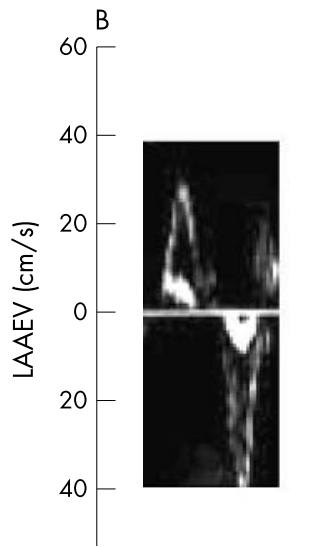

RAA

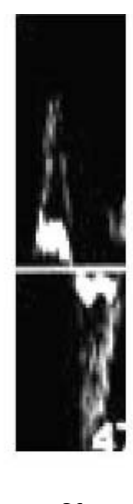

CS

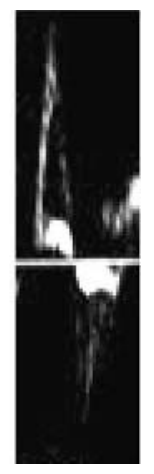

$\mathrm{BIA}$

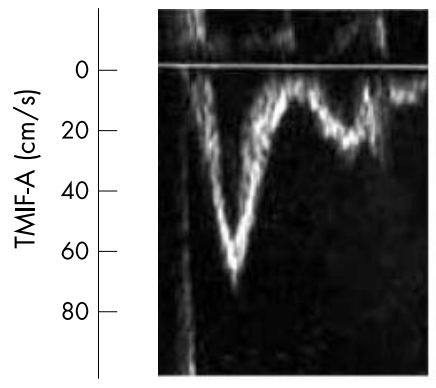

RAA

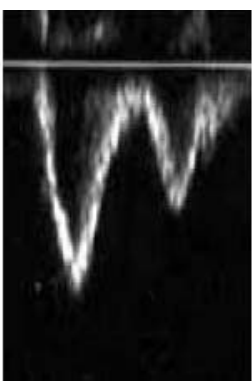

CS

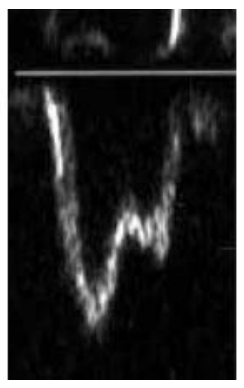

BIA

Figure 5 (A) Comparison of LAAEV, LAAEF, TMIF-A, and SEC between right atrial appendage (RAA), lateral coronary sinus (CS), and biatrial pacing (BIA). ${ }^{*} p=0.001,{ }^{* *} p<0.001,{ }^{* * *} p=0.0001,{ }^{* * * *} p<0.0001$, RAA $v$ BIA. (B) Representative LAAEV. (C) Representative TMIF-A.

remaining nine had no structural heart disease (table 1). Mean (SD) left ventricular ejection fraction was 50.8 (13.3)\% and left atrial size was $45.2(6.1) \mathrm{mm}$. All patients were successfully cardioverted as defined by the maintenance of sinus rhythm. After cardioversion, sinus rates were under 80 beats/min (64.7 (13.0) beats/min) in all patients. No patient had an early AF relapse for about two hours after successful cardioversion during this study protocol. Before cardioversion, 16 of 20 patients $(80 \%)$ were found to have SEC.

\section{Atrial mechanical function during sinus rhythm after cardioversion of persistent AF}

After cardioversion, LAAEV (fig 1) and LAAEF (fig 2) decreased compared with the pre-cardioversion value. LAAEV decreased from $28.4(17.7) \mathrm{cm} / \mathrm{s}$ to $14.6(10.1) \mathrm{cm} / \mathrm{s}$ $(\mathrm{p}<0.001)$, whereas LAAEF decreased from $21.7(12.0) \%$ to $13.8(8.5) \%(\mathrm{p}=0.001)$.

After electrical cardioversion, new or increased SEC was detected in 18 of the 20 patients (90\%). SEC grade increased from $1.4(1.2)$ to $2.6(1.0)(\mathrm{p}<0.001)$ (fig 3).

\section{Effect of atrial pacing rate and site on atrial mechanical function}

Compared with sinus rhythm, atrial pacing at 80 beats/min significantly increased LAAEV from $14.6(10.1) \mathrm{cm} / \mathrm{s}$ to 33.4 (19.8) cm/s ( $\mathrm{p}=0.001$ ) (fig 1$)$, increased LAAEF from 13.8
(8.5)\% to $32.1(11.2) \%(\mathrm{p}<0.001)$ (fig 2$)$, increased TMIF-A from 24.6 (11.9) cm/s to $45.6(21.0) \mathrm{cm} / \mathrm{s}(\mathrm{p}<0.001)$ (fig 4$)$, and reduced SEC grade from 2.6 (1.0) to $1.6(0.9)$ $(\mathrm{p}<0.001)$ (fig 3$)$. An increase in the atrial pacing rate resulted in significantly greater increases in LAAEV from 33.4 (19.8) cm/s to $55.0(23.6) \mathrm{cm} / \mathrm{s}(\mathrm{p}<0.0001)$ and in LAAEF from $32.1(11.2) \%$ to $47.7(11.4) \%(\mathrm{p}<0.0001)$ at 80 beats/ $\min v 120$ beats/min, respectively) (figs 1 and 2). Increased atrial pacing at 80 beats/min $v 120$ beats/min also produced a significant reduction in the magnitude of SEC from $1.6(0.9)$ to $0.5(0.8)(\mathrm{p}<0.0001)$ (fig 3). Comparing between pacing sites, biatrial pacing resulted in the most significant improvement in atrial function (LAAEV, $33.2(19.3) \mathrm{cm} / \mathrm{s} v$ $53.7(23.9) \mathrm{cm} / \mathrm{s}, \mathrm{p}=0.0001$; LAAEF, 31.9 (11.1)\% $v 46.2$ (12.6)\%, p < 0.0001; TMIF-A, $37.7 \quad(18.3) \mathrm{cm} / \mathrm{s} \quad v \quad 54.1$ $(21.2) \mathrm{cm} / \mathrm{s}, \mathrm{p}<0.001$; SEC grade, 1.4 (1.1) $v 0.8$ (0.9), $\mathrm{p}=0.001$, with RAA $v$ biatrial pacing) (fig 5 ). There was no significant difference in these parameters between RAA and coronary sinus pacing.

There was no significant difference in interobserver nor intraobserver variability.

\section{Maximum $P$ wave duration during sinus rhythm and atrial pacing}

After conversion to sinus rhythm, all patients had prolonged $\mathrm{P}$ wave duration (135 (15) ms). There was no significant 
change in the duration of the $\mathrm{P}$ wave between sinus rhythm, RAA pacing ( 141 (23) ms), and coronary sinus pacing (147 (38) $\mathrm{ms}$ ). However, biatrial pacing resulted in a $\mathrm{P}$ wave with the shortest duration (102 (13) ms, p $<0.001)$.

\section{DISCUSSION \\ Main findings}

The present study provides new findings about atrial mechanical function in mechanically stunned atrium after cardioversion of persistent AF. Atrial pacing results in an acute improvement in atrial mechanical function just after cardioversion of persistent AF. This effect of atrial pacing has a positive force-frequency relation. The most significant improvement in atrial mechanical function was achieved by simultaneous biatrial pacing when compared with single site atrial pacing.

\section{Atrial mechanical function after conversion to sinus rhythm}

We observed a high incidence of stunned left atrial and LAA function after electrical cardioversion of persistent $\mathrm{AF}$ in agreement with previous reports. ${ }^{1-9}$ No significant difference in the incidence and severity of stunned atrial mechanical function after all modes of cardioversion of AF has been reported..$^{313}{ }^{17}$ Therefore, the decrease in LAAEV and LAAEF and new or increased SEC we describe in this study may be typical findings after conversion to sinus rhythm.

\section{Effect of atrial pacing rate}

It has been reported that ventricular contraction is greater with increasing heart rate in the normal heart, but not in the failing heart. ${ }^{18}$ The release of calcium from sarcoplasmic storage is thought to be the mechanism underlying the frequency dependent force generation. ${ }^{19} 20$ It remains unknown whether atrial mechanical function is influenced by an increased heart rate in the normal human heart. Agmon and colleagues ${ }^{21}$ reported a positive relation between heart rate and LAAEV, but patients with sinus tachycardia had a worse LAAEV. Sanders and colleagues ${ }^{14} 15$ showed in patients after cardioversion of chronic atrial flutter and short duration AF that LAAEV improved stepwise with an increased pacing rate at atrial cycle lengths between $750 \mathrm{~ms}$ and $500 \mathrm{~ms}$. In the present study, we also observed a stepwise improvement in stunned LAA mechanical function with increased pacing rate after cardioversion of persistent AF. This suggests that in patients with atrial stunning after cardioversion of persistent AF, the atrial contractile apparatus is intact but functionally impaired and suggests also that atrial stunning is reversible and may be associated with a different mechanism that causes heart failure. Atrial mechanical dysfunction after atrial tachyarrhythmias is related to a diminished calcium transient, ${ }^{22-24}$ resulting from a reduction in L-type calcium current density. ${ }^{25}$ In the present study we suggest that increased intracellular calcium concentrations associated with increasing atrial pacing may improve atrial mechanical function and that a deficiency in intracellular calcium may be responsible for atrial stunning after cardioversion of persistent AF.

\section{Effect of atrial pacing for global left atrial function}

A previous report about the effect of pacing on atrial mechanical function was limited to the evaluation of LAA function. ${ }^{14}{ }^{15}$ Agmon and colleagues ${ }^{27}$ showed that LAAEV correlates poorly with global left atrial variables, indicating that LAAEV is inadequate to be used as a surrogate of global left atrial function. In the present study, we have shown that atrial pacing significantly increased TMIF-A. To the best of our knowledge, this is the first clinical report that atrial pacing can improve global left atrial function in the mechanically stunned atrium after cardioversion of persistent AF.

\section{Effect of atrial pacing site}

Recently, it has been shown that biatrial pacing can be more effective than standard RAA pacing in reducing the recurrence of $\mathrm{AF}^{28-32}$ Acute electrophysiological studies have suggested that a reduction in the atrial conduction delay by simultaneous activation of both atria is an important mechanism of improved prevention of AF. ${ }^{28} 29313334$ The effects of alternate and multisite atrial pacing on atrial stunning after cardioversion of AF have yet to be evaluated. In the present study biatrial pacing produced a significant improvement in atrial mechanical function after cardioversion of persistent AF in comparison with single site (RAA or coronary sinus) atrial pacing. Patients with a prolonged $\mathrm{P}$ wave on the ECG are thought to have intra-atrial conduction delay and atrial dyssynchrony. ${ }^{35}$ In the present study, all patients had a prolonged $\mathrm{P}$ wave after conversion to sinus rhythm. P waves of the shortest duration were obtained after biatrial pacing compared with sinus rhythm, RAA pacing, and coronary sinus pacing. We suggest that intra-atrial resynchronisation by biatrial pacing may be important in producing the most improvement in atrial mechanical function compared with single site atrial pacing.

\section{Clinical implications}

Atrial mechanical stunning is thought to be one of the mechanisms responsible for the increased risk of thromboembolic complications after cardioversion of AF. ${ }^{56}$ Our findings suggest that atrial pacing just after cardioversion of persistent AF can potentially prevent subsequent thromboembolic complications. The observation that atrial pacing significantly reversed TMIF-A implicates an improvement in the haemodynamic function after cardioversion of AF. The finding that pacing rate may be an important factor in increasing atrial mechanical function may also have implications in the choice of post-cardioversion pacing rates in patients with implanted devices. The present study showed that biatrial pacing may be the best way to achieve these benefits. In clinical practice, biatrial pacing just after cardioversion of persistent AF from temporary leads inserted into the RAA and coronary sinus would be recommended.

\section{Limitations}

There are several limitations in the present study. Firstly, atrial pacing did not exceed 120 beats/min. In the clinical setting, we believe that atrial pacing over 120 beats/min may be inconvenient because patients often experience palpitation, decreased ventricular filling time, and impaired haemodynamic function in some patients, and may re-induce AF. Secondly, we could not evaluate the effects of the pacing rate on global left atrial function. Because the transmitral inflow pattern at 100 beats/min and 120 beats/min indicates a single fusion wave, consisting of both the early filling and atrial filling waves at all pacing sites, we could not evaluate TMIF-A at either 100 beats/min or 120 beats/min. Whether the effect of atrial pacing on global left atrial function has a positive force-frequency relation is still unknown. Thirdly, although we have shown the acute benefits of atrial pacing on atrial mechanical function just after electrical cardioversion of persistent AF, it remains unknown whether the improvement is sustained during continuous pacing over several weeks and whether the effects would remain over the time course of the recovery of atrial mechanical function. Unfortunately, at present, these are extremely limited because of the requirement for long term implanted devices. These may be important issues for further research. 


\section{Conclusion}

Atrial pacing at increased rates can improve atrial mechanical function after cardioversion of persistent AF. Biatrial pacing might be the most effective technique to reverse atrial mechanical stunning.

\section{Authors' affiliations}

M Takagi, A Doi, N Shirai, K Hirata, Y Takemoto, K Takeuchi,

J Yoshikawa, Department of Internal Medicine and Cardiology, Osaka City University Graduate School of Medicine, 1-4-3 Asahimachi, Abeno-ku, Osaka 5458585, Japan

\section{REFERENCES}

1 Grimm RA, Stewart WJ, Maloney JD, et al. Impact of electrical cardioversion for atrial fibrillation on left atrial appendage function and spontaneous echo contrast: characterization by simultaneous transesophageal echocardiography. J Am Coll Cardiol 1993;22:1359-66.

2 Fatkin D, Kuchar DL, Thorburn CW, et al. Transesophageal echocardiography before and during direct current cardioversion of atrial fibrillation: evidence for "atrial stunning" as a mechanism of thromboembolic complications. J Am Coll Cardiol 1994;23:307-16.

3 Grimm RA, Leung DY, Black IW, et al. Left atrial appendage "stunning" after spontaneous conversion of atrial fibrillation demonstrated by transesophageal Doppler echocardiography. Am Heart J 1995;130:174-6.

4 Antonielli E, Pizzuti A, Bassignana A, et al. Transesophageal echocardiographic evidence of more pronounced left atrial stunning after chemical (propafenone) rather than electrical attempts at cardioversion from atrial fibrillation. Am J Cardiol 1999;84:1092-6.

5 Manning WJ, Leeman DE, Gotch PJ, et al. Pulsed Doppler evaluation of atrial mechanical function after electrical cardioversion of atrial fibrillation. J Am Coll Cardiol 1989:13:617-23.

6 Black IW, Fatkin D, Sagar KB, et al. Exclusion of atrial thrombus by transesophageal echocardiography does not preclude embolism after cardioversion of atrial fibrillation: a multicenter study. Circulation 1994;89:2509-13.

7 Falcone RA, Morady F, Armstrong WF. Transesophageal echocardiographic evaluation of left atrial appendage function and spontaneous contrast formation after chemical or electrical cardioversion of atrial fibrillation. Am J Cardiol 1996;78:435-9.

8 Omran $\mathrm{H}$, Jung W, Rabahieh $\mathrm{R}$, et al. Left atrial chamber and appendage function after internal atrial defibrillation: a prospective and serial transesophageal echocardiographic study. J Am Coll Cardiol 1997;29:131-8.

9 Harjai KJ, Mobarek SK, Cheirif J, et al. Clinical variables affecting recovery of left atrial mechanical function after cardioversion from atrial fibrillation. J Am Coll Cardiol 1997:30:481-6.

10 Louie EK, Liu D, Reynertson SI, et al. Stunning of the left atrium after spontaneous conversion of atrial fibrillation to sinus rhythm: demonstration by transesophageal Doppler techniques in a canine model. J Am Coll Cardiol 1998;32:2081-6.

11 Harjai KJ, Mobarek SK, Abi-Samra F, et al. Mechanical dysfunction of the left atrium and the left atrial appendage following cardioversion of atrial fibrillation and its relation to total electrical energy used for cardioversion. Am J Cardiol 1998;81:1125-9.

12 Spark PB, Jayaprakash S, Mond HG, et al. Left atrial mechanical function after brief duration atrial fibrillation. J Am Coll Cardiol 1999;33:342-9.

13 Zipes DP. Atrial fibrillation: a tachycardia-induced atrial cardiomyopathy. Circulation 1997;95:562-4.

14 Sanders P, Morton JB, Morgan JG, et al. Reversal of atrial mechanical stunning after cardioversion of atrial arrhythmias: implications for the mechanisms of tachycardia-mediated atrial cardiomyopathy. Circulation 2002; 106:1806-13

15 Sanders P, Morton JB, Kistler PM, et al. Reversal of atrial mechanical dysfunction after cardioversion of atrial fibrillation: implications for the mechanisms of tachycardia-mediated atrial cardiomyopathy. Circulation 2003; 108:1976-84.

16 Fatkin D, Kelly RP, Feneley MP. Relations between left atrial appendage blood flow velocity, spontaneous echocardiographic contrast and thromboembolic risk in vivo. J Am Coll Cardiol 1994;23:961-9.

17 Shapiro EP, Effron MB, Lima S, et al. Transient atrial dysfunction after conversion of chronic atrial fibrillation to sinus rhythm. Am J Cardiol 1988;62:1202-7

18 Mulieri LA, Hasenfuss G, Leavitt B, et al. Altered myocardial force-frequency relation in human heart failure. Circulation 1992:85:1743-50.

19 Brixius $\mathrm{K}$, Pietsch $\mathrm{M}$, Schwinger $\mathrm{RH}$. The intracellular $\mathrm{Ca}(2+)$-homeostasis influences the frequency-dependent force-generation in man. Basic Res Cardiol 1999;94:152-8

20 Hasenfuss G, Reinecke $H$, Studer R, et al. Relation between myocardial function and expression of sarcoplasmic reticulum $\mathrm{Ca}(2+)$-ATPase in failing and nonfailing human myocardium. Circ Res 1994;75:434-42.

21 Agmon Y, Khandheria BK, Meissner I, et al. Left atrial appendage flow velocities in subjects with normal left ventricular function. Am J Cardiol 2000;86:769-73.

22 Sun H, Gaspo R, Leblanc N, et al. Cellular mechanisms of atrial contractile dysfunction caused by sustained atrial tachycardia. Circulation 1998;98:719-27

23 Ausma J, Duimel $\mathrm{H}$, Thone $\mathrm{F}$, et al. Changes in ultrastructural calcium distribution in goat atria during atrial fibrillation. J Mol Cell Cardiol 2000;32:355-64.

24 Yue L, Feng J, Gaspo R, et al. lonic remodeling underlying action potential changes in a canine model of atrial fibrillation. Circ Res 1997:81:512-25.

25 Van Wagoner DR, Pond AL, Lamorgese M, et al. Atrial L-type Ca2+ currents and human atrial fibrillation. Circ Res 1999;85:428-36.

26 Brundel BJJM, Gelder ICV, Henning RH, et al. Gene expression of proteins influencing the calcium homeostasis in patients with persistent and paroxysmal atrial fibrillation. Cardiovasc Res 1999;42:443-54

27 Agmon Y, Khandheria BK, Meissner I, et al. Are left atrial appendage flow velocities adequate surrogates of global left atrial function? A populationbased transthoracic and transesophageal echocardiographic study. J Am Soc Echocardiogr 2002;15:433-40.

28 Daoud EG, Dabir R, Archambeau M, et al. Randomized, double-blind trial of simultaneous right and left atrial epicardial pacing for prevention of post-open heart surgery atrial fibrillation. Circulation 2000;102:761-5.

29 Fan K, Lee KL, Chiu CS, et al. Effects of biatrial pacing in prevention of postoperative atrial fibrillation after coronary artery bypass surgery. Circulation 2000;102:755-60.

30 Gerstenfeld EP, Hill MR, French SN, et al. Evaluation of right atrial and biatrial temporary pacing for the prevention of atrial fibrillation after coronary artery bypass surgery. J Am Coll Cardiol 1999;33:1981-8.

31 Leclercq JF, De Sisti A, Fiorello $P$, et al. Is dual site better than single site atrial pacing in the prevention of atrial fibrillation? Pacing Clin Electrophysiol 2000;23:2101-7

32 Ramdat MA, Beukema WP, Oude LH. Multisite or alternate site pacing for the prevention of atrial fibrillation. Am J Cardiol 1999;83:237-40.

33 Papageorgiou P, Monahan K, Boyle NG, et al. Site-dependent intra-atrial conduction delay: relationship to initiation of atrial fibrillation. Circulation 1996;94:384-9.

34 Yu WC, Chen SA, Tai CT, et al. Effects of different atrial pacing modes on atrial electrophysiology: implicating the mechanism of biatrial pacing in prevention of atrial fibrillation. Circulation 1997:96:2992-6.

35 Centurion OA, Isomoto S, Fukatani M, et al. Relationship between atrial conduction defects and fractionated atrial endocardial electrograms in patients with sick sinus syndrome. Pacing Clin Electrophysio 1993; 16:2022-33. 